Kompass

Dermatologie

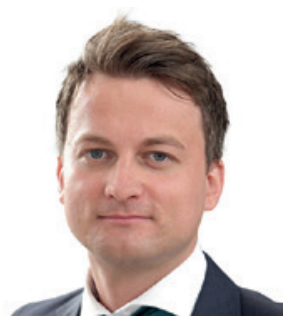

\section{David Matusiewicz}

FOM Hochschule für Oekonomie \& Management gemeinnützige Gesellschaft mbH, KCG KompetenzCentrum für Management im Gesundheits- und Sozialwesen, Essen, Deutschland

\title{
Wir werden uns an den «Hautarzt in der Hosentasche» gewöhnen müssen
}

Die Digitale Transformation im Gesundheitswesen wird die Medizin in den nächsten Jahren deutlich verändern. Und dies betrifft Diagnostik, Therapie und Nachsorge. Es wird Marktverschiebungen von stationär zu ambulant und von ambulant nach Hause geben. Der Patient wird mit den FüBen abstimmen und wie in anderen Branchen den Dienstleister bevorzugen, der ihm die schnellste Lösung mit größter Qualität liefern wird. Für einige Dermatologen klingt das wohl nach Dystopie, für einige Start-Ups und die Patienten nach Utopie. Ich habe mich bereits während meiner Doktorarbeit bei Jürgen Wasem am Lehrstuhl für Medizinmanagement in Essen im Jahr 2012 mit Big Data in der Dermatologie beschäftigt. Genauer mit der Versorgungssituation bei der juvenilen Psoriasis mit einem Datensatz von knapp 7 Mio. Versicherten - und mir wurde damals schon klar, wie wichtig Daten für die Dermatologie sind. Seitdem ist viel passiert und die Dermatologie ist für mich innerhalb der Medizin neben der Radiologie - die Disziplin, die maßgeblich von der Digitalisierung profitieren wird. Warum ist das so? Die Dermatologie ist eine «sehende» und «sprechende» Medizin. Beide Bereiche sind prädestiniert für das Nutzen von digitalen Entwicklungen, die Mithilfe von neuronalen Netzen, maschinellem Lernen und schließlich künstlicher Intelligenz. Heute bereits ist die Smartphone-Kamera gut genug, um Bilder in einer ausreichenden Qualität von der Haut aufzunehmen und mit entsprechenden digitalen Entscheidungsunterstützungssystemen (EUS) zu interpretieren. Das digitale Vieraugenprinzip (Zentaur-Modell aus dem analogen Dermatologen und einem digitalen Helfer) ist heute bereits in der Versorgung angekommen. Die Beispiele in dem vorliegenden Heft zeigen bereits eindrucksvoll was heute schon möglich ist.

Die Teledermatologie ist auf dem Vormarsch. Zudem kann der Patient heute jederzeit eine erste Einschätzung zu bspw. einer Auffälligkeit auf seiner Haut anstoßen und das jetzt sofort - egal wann jetzt sofort ist (auch irgendwann nachts). Im Anschluss erhält er ein schriftliches Feedback. Auch wenn die digitale Lösung heute noch als «Second-Best»-Lösung gesehen wird, so ist diese auch wichtig, um Fälle vorzusortieren und damit Fälle, die keine Fälle sind, auszusortieren. Das spart Ressourcen für unser Gesundheitssystem mit durchschnittlich 18 Arztkontakten pro Jahr.

Ich versuche auch immer die Patientenperspektive einzunehmen, denn für diesen stellt sich der Nutzen am höchsten dar. Als Patient wünsche ich mir, dass ich regelmäßig (ggfs. mehrmals im Jahr) zuhause oder in einer Kabine bspw. im Fitnessstudio oder Supermarkt (wie es die MinuteClinic als Einzelhandelsklinik in den USA bereits anbietet) ein Hautkrebsscreening machen kann, bei dem ich unmittelbar ein Ergebnis erhal- te und nur im Verdachtsfall einen Dermatologen aufsuchen muss. Früher sind die Menschen zur Sonnenbank gegangen, morgen gehen Sie zum Hautscan als Lifestyle-Produkt.

Als Patient wünsche ich mir zudem schnellen Zugang zur telemedizinischen Gesprächen (per Video oder Chatbot) und asynchrone Kommunikation mit meinem Dermatologen, aber auch weiterhin die Möglichkeit face-to-face mit ihm sprechen zu können. Und dafür bin ich bereit out-ofpocket als Selbstzahler auch etwas zu bezahlen. Durch Digitale Gesundheitsanwendungen (DiGA) werden aber auch zum Ende des Jahres womöglich erste Anwendungen für den ersten Gesundheitsmarkt vorbereitet und das wird die Versorgung weiter digital verändern.

Die Digitalisierung ist keine Gefahr für den Arzt, sie ist gleichwohl ein machtvolles Instrument, das in den nächsten Jahren zunehmend den Versorgungsalltag ergänzen wird. Wir werden uns an den Hautarzt «in der Hosentasche» gewöhnen müssen. Ob es dem Dermatologen passt oder nicht. Der Kunde wird darüber entscheiden. Ich wünsche Ihnen eine spannende Lektüre,

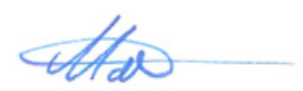

Ihr Prof. Dr. David Matusiewicz information@karger.com @ (c) 2020 S. Karger GmbH, Freiburg www.karger.com/kkd

Karger"
Prof. Dr. David Matusiewicz

FOM Hochschule für Oekonomie \& Management gemeinnützige Gesellschaft mbH Herkulesstr. 32, 45127 Essen, Deutschland david.matusiewicz@fom.de 